\title{
Molecular dynamics of acetate-based ionic liquids
}

\author{
RODOLFO R HOLANDA ${ }^{1}$, DHEIVER F SANTOS ${ }^{1,5} \mathbb{D}^{\mathbb{D}}$, TATIANA G D DA SILVA ${ }^{1}$, \\ SHAIK BABU ${ }^{3, * \mathbb{D}}$, CESAR C SANTANA ${ }^{2}$ and WALISSON J SOUZA ${ }^{4}$ \\ ${ }^{1}$ Engenharia de Petróleo, Centro Universitário Tiradentes, Maceió, AL, Brazil \\ ${ }^{2}$ Programa de pós-graduação em Engenharia de Processos, Universidade Tiradentes, Aracaju, SE, Brazil \\ ${ }^{3}$ Department of Physics, Koneru Lakshmaiah Education Foundation, Guntur 522 502, Andhra Pradesh, India \\ ${ }^{4}$ Faculdade Pio Décimo, Engenharia Civil, Aracaju, SE, Brazil \\ ${ }^{5}$ Engenharia Mecatrônica, Centro Universitário Tiradentes - Unit/Al, Av. Gustavo Paiva, 5017 - Cruz das Almas, Maceió, \\ AL CEP 57038-000, Brazil \\ *Author for correspondence (babu.computers@gmail.com)
}

MS received 1 June 2018; accepted 16 July 2018; published online 27 March 2019

\begin{abstract}
A radial distribution analysis of pairs between the constituent atoms of protic ionic 2-(hydroxy)ethylammonium acetate was performed with molecular dynamics technique (MDT). The ionic liquid structure will help us in different engineering applications, as emulsification process (petroleum industry). The results presented here show that the development of force field to predict the high interaction behaviour between the cation and the anion, as well as the formation of ion-pair dispersed aggregates. These results can contribute to help the different applications of ionic liquids.
\end{abstract}

Keywords. Ionic liquids; molecular dynamics; RDF.

\section{Introduction}

Ionic liquids have attracted attention from the scientific community, such as electrolytes or proton donors in fuel cells, or in oil-water separation processes, due to their thermodynamic, transport and electrochemical properties. Over the years, there have been improvements in their thermal and electrochemical properties and the use of fuel cells with ionic liquids based on the ammonium ion, called protic ionic liquid. The molecular dynamics study gives us information about the structure (salt liquid phase), based on the ammonium ion. The levels of interactions between cation and anion, discussion of ion size and structural form is an important factor for the process of charge transfer, besides the possibility of helping the different physicochemical properties.

Some works have pointed out that the molecular dynamics studies of ionic liquids allow one to understand the molecular characteristics of ionic liquids, and their interactions. The works of Fumino et al [1]; Herrera et al [2]; Shimizu et al [3]; Nasrabadi and Gel [4] provide detailed information about their properties and showing the main molecular characteristics, defining their structural, energy and dynamic properties of their constituent atoms. Fumino et al [1] used infrared spectroscopy to observe the molecular interactions that occurred in the ionic liquid. The authors have shown that the IL (ionic liquid) structure is largely determined by Coulomb energy, dispersion forces and hydrogen bonds between species. Studies also show numerous ionic-pairs isolated (ionic-pairs are structures where there are basically ions attached to one another and these structures are isolated inside ionic liquids).
Shimizu et al [3] studied the properties of a series of ionic liquids based on perfluorobutylsulphonate and through structure factor calculations, along the chain augmentation and by fixing the anion, the different IL (ionic liquids) grow continuously in terms of size, shape and degree compression.

The authors also observed different levels of partial ionic liquid aggregation. Herrera et al [2] reported a study of molecular dynamics of ionic liquids based on glycine, serine, alanine and phenyl alanine anions. The authors report a large cation-anion interaction close to $2 \AA$ and that the energies of interactions are similar when we compare cations, i.e., the anion effect is more significant than the structural effect produced by the cation alteration. Nasrabadi and Gel [4] analysed the structure and transport properties of a series of ionic liquids based on ammonium triflate. The authors have described in detail that the anion preferably approaches the cation to form hydrogen bonds between oxygen sulphonate atoms and the amino acid hydrogen.

The novelty of this work is theoretical and computational study of the effect of levels of interactions, relative distances, and energies between the atoms in the network on the radial distributions of pairs of atoms for the ionic liquid 2-(hydroxy)ethylammonium acetate, where these results are essential to deal with different applications of ionic liquids, as emulsification process realized in the petroleum industry.

Thus, the present work aims to observe quantitatively through the analysis of radial distribution of pairs of atoms, the different levels of interactions and the relative distances 
and energies between the atoms in the network using for the technique of molecular dynamics, for the ionic liquid 2-(hydroxy)ethylammonium acetate.

\section{Methodology}

\subsection{Molecular dynamics}

Simulations of molecular dynamics using NPT statistical assemblies with a time step of $1 \mathrm{fs}$ were performed to determine the density of pure components. For each pure component of ionic liquid, a system consisting of 100 ion pairs was used. To join the system ions in a cubic simulation box, the Playmol software was used to reach a density of $1.0-1.1 \mathrm{~g} \mathrm{~cm}^{-3}$. With Playmol computer software to create a first point for MD simulations by packing particles in defined regions of space. The packing guarantees that short range repulsive interactions do not disrupt the simulations. The simulator housings were first heated from 298 to $500 \mathrm{~K}$ with a 1 fs step time over 25 ps using a Nosé-Hoover chain thermostat to avoid the presence of local energies. Then, the box was gradually cooled to a temperature of $298 \mathrm{~K}$ for $300 \mathrm{ps,}$ thermally being pressurized to $298 \mathrm{~K}$ for $2 \mathrm{~ns}$. Once cooled, the NPT ensemble and Nosé-Hoover barostat with a coupling time of 1000 fs were employed to balance the density using a set point of $1 \mathrm{~atm}$ pressure. The densities proposed in the system converged around $2 \mathrm{~ns}$ and a sampling time of at least $10 \mathrm{~ns}$ was used to determine the density average in finished simulations. The simulations were performed using a cluster with a processing capacity of 20 teraflops, and $110 \mathrm{~TB}$ of data storage.

The force field $E(r)$ is described as the sum of several energy contributions, including bound contributions (bounding lengths and angles, dihedral angles) and terms for unbound atoms (van der Waals and Coulomb interactions).
The CHARMM force field is represented by equation (1) and is used by this work:

$$
\begin{aligned}
E= & \sum K b\left(b-b_{0}\right)^{2}+\sum \theta\left(\theta-\theta_{0}\right)^{2} \\
& +\sum \frac{K \phi}{2}\left[\left(1+\cos \left(n \phi-\phi_{0}\right)\right)\right] \\
& +\sum \frac{K \lambda}{2}\left[\left(1+\cos \left(n \lambda-\lambda_{0}\right)\right)\right] \ldots \ldots . . \\
& +\sum\left\{\left[\left(\frac{R_{i j}}{r_{i j}}\right)^{12}-2\left(\frac{R_{i j}}{r_{i j}}\right)^{6}+\frac{q_{i} q_{j}}{r_{i j}}\right]\right\} .
\end{aligned}
$$

\subsection{Sampling of atoms}

Sampling of atoms is done in the simulation box in different states to obtain the radial distribution of pairs. The radial function is a level measure of correlation between the particles of a system of many atoms. It is represented by $g(r)$ and represents the structural characteristics and intensity of interaction levels between atoms previously defined in the sampling system at a given time of integration. It can be interpreted as a 'local density', i.e., when $g(r)$ tends to 1 , the density is the average of the system. When $g(r)$ presents values greater than the means, the local density is higher than the system average, while lower values of $g(r)$ show that the density is lower than the sampled system average. Further details on the radial distribution function can be found in the works of Perez-Blanco and Maginn [5]; Perkins et al [6]; Margulis et al [7]; and Yee et al [8].

\subsection{Chemical structure of ionic liquid}

Figure 1 presents details about the ionic liquid used in this work.

\section{Results and discussion}

The validation of the force field developed by this work consists in comparing the experimental density values obtained in the laboratory and the density obtained through the

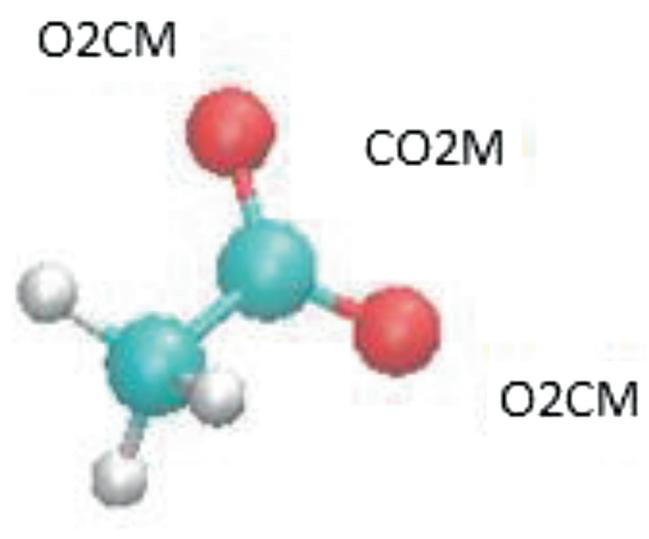

NRP

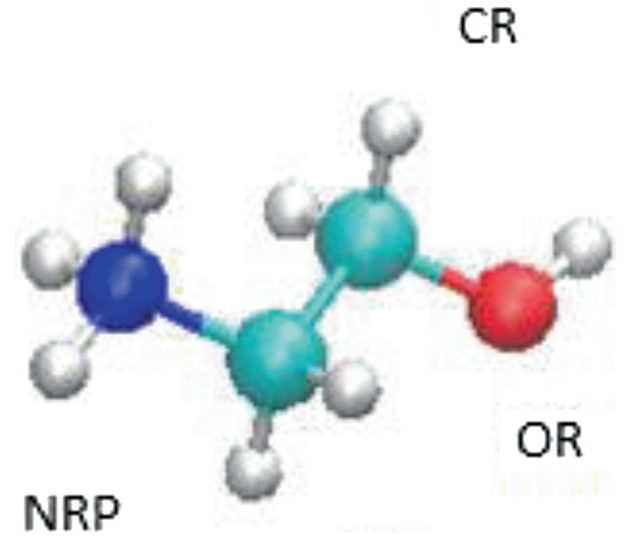

CR

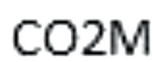

Figure 1. Molecular structure of the ionic liquid 2-(hydroxy)ethylammonium acetate. 

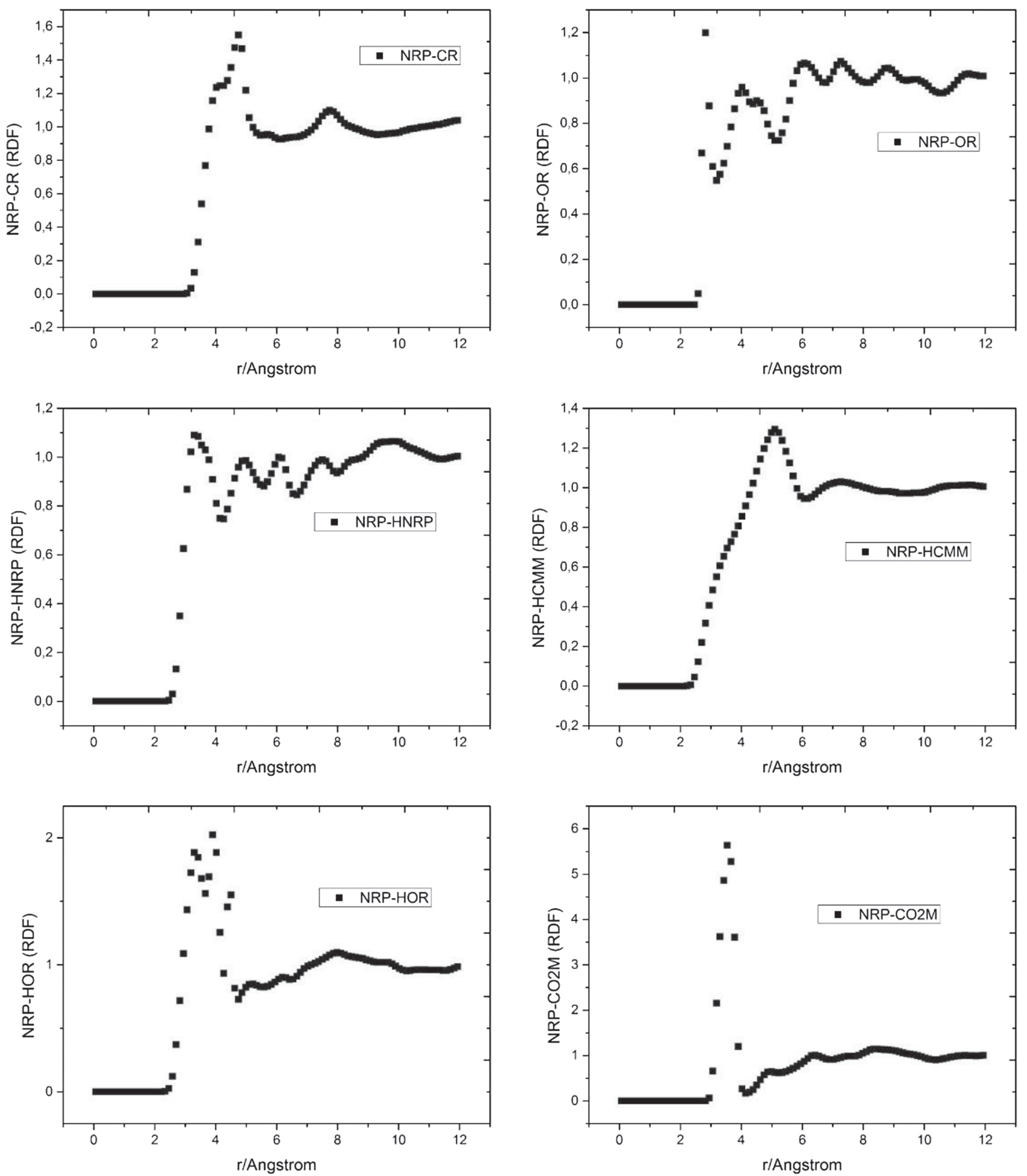

Figure 2. The radial distribution function (RDF) between nitrogen and carbon (NRP-CR). Showing the interaction between cations. Nitrogen and oxygen (NRP-OR). Nitrogen and cation hydrogen (NRP-HNRP). Nitrogen and hydrogen (NRP-HCMM). Nitrogen and hydrogen (NRP-HOR). Nitrogen and carbon (NRP-CO2M). Introducing the interaction between cation-anion.

computational simulations. When the experimental data approximate quantitatively with the simulated data, the force field is reported as valid. In this work, the experimental data corroborate with the simulated data.
Figure 2 shows the radial distribution function layers solvation is centered in the NRP atom of the cation. It is observed that the existence of two peaks, one is well defined and one is not. The first peak refers to the first 
hydration layer of NRP atom and the carbon atoms of the cation and extends by 2.6-4.5 $\AA$ with maximum $4.5 \AA$. After $8 \AA$ there is a total separation between the atoms of NRP$\mathrm{CR}$ cations. There is a possibility of formation of hydrogen bonds between cations. These hydrogen bonds are responsible for the changes of the geometrical parameters involving the NRP atom and consequently, changes in the organization of the cations in the network. The second peak has very small intensity to indicate the formation of a second overlapping layer.

Figure 2 also shows the radial distribution function of the multiple layers of solvation layers centered at the NRP atom of the cation. It is observed the existence of several peaks, one is well defined and the other is not so well defined. The first peak refers to the first hydration layer of the NRP atom in cation, in well-defined 2-3 $\AA, 2-3 \AA$ also at maximum. From the start of the first layer until $12 \AA$ there are no separation solvation between the NRP-OR atom cations. There is a possibility of formation of hydrogen bonds between cations. These hydrogen bonds are responsible for the changes of the geometrical parameters involving the NRP atom and consequently, changes in the organization of the cations in the network. The solvation layer between 2.5 and $4.5 \AA$ carries the largest number of atoms.. With increasing distance, the intensity of the population of reference atoms tends to decrease and also decreases the interaction between them as well.

The radial distribution function of the multiple layers of solvation centered in NRP atom, it is observed the existence of several peaks, none so well-defined. The first peak refers to the first hydration layer NRP atom cations by hydrogen atoms bound to nitrogen in $2.5 \AA, 2.5 \AA$ at maximum also. From the start of the first layer until $12 \AA$, there are no separate solvations between the NRP-HNRP atoms cations. There is a possibility of formation of hydrogen bonds between cations. The solvation layer between 2.5 and $4 \AA$ contains the largest number of atoms in the figure presented. Peaks may overlap, interfering with the analysis of the different layers of solvation. One possibility is the speed of the hydrogen atom being higher than the other atoms.

The radial distribution function of a solvation layer centered in NRP atom of the cation, it is observed the existence of a peaks, one well-defined and the other not so well-defined, i.e., a thin layer of solvation. The first peak refers to the first hydration layer NRP atom by hydrogen atoms present in the carbon cation defined between 2.3 and $6 \AA$ with maximum 4.5 $\AA$, this hydration layer comprises larger number of atoms of type NRP-HCMM. From the start of the first layer until $12 \AA$ there is total separation solvation between the NRP-HCMM atom cations. There is a possibility of formation of hydrogen bonds between cations.

Figure 2 also shows the radial distribution function layers solvation centered NRP atom cation. It is observed the existence of two well-defined peaks. The first peak refers to the first hydration layer NRP atom by oxygen atoms of the cation, in well-defined $2.3 \AA, 2.3 \AA$ also at maximum. The second peak refers to the second hydration layer NRP atom by oxygen atoms of the cation and defined in $4 \AA$, $4 \AA$ also at maximum. From the beginning of the second layer to solvation $12 \AA$, there is total separation between NRP-HOR atom cations. The probability of finding a nitrogen atom with high interaction with hydrogen from the cation oxygen is greater in the range of $2-4 \AA$.

Also, the radial distribution function of the single layers of solvation centered NRP atom of the cation, the existence of a well-defined peak is observed. The peak refers to the first hydration shell of the atom by anion NRP carbon atoms, well defined in $3 \AA$, with a maximum at $3 \AA$ too. From the beginning of the first layer to solvation $12 \AA$, there is total separation between NRP-CO2M atom cations. There is a possibility of hydrogen bonding between cation-anion. These hydrogen bonds are responsible for the changes of the geometrical parameters involving the NRP atom, consequently, charges in the organization of the cation in the network. The solvation layer between 2.5 and $4 \AA$ carries the highest number of atoms. As the density increases, the intensity of the population of reference atoms tends to decrease. The probability of finding a nitrogen atom with high carbon interaction of the oxygen of the anion is greater in the range of $2.5-4 \AA$.

In general, we have a density corresponding to the liquid phase, the function $g(r)$ still shows a certain ordering of the system, with its several layers of solvation. The results obtained showed a great deal of similarity in behaviour of ionic vs. fluids of the literature $[9,10]$. Analysing the radial function of the liquid in figure 2 , we see different maxima, relative to the interaction between the selected atoms, and a considerable increase in some maxima relative to the thickness of solvation layer, are also presented. The results show a preferred path between cation-cation and its multiple layers, and when analysing cation-anion, the system presents a separation between the selected atoms.

\section{Conclusion}

The molecular dynamics was used to analyse the atomic structure of the ionic liquid 2-(hydroxy)ethylammonium acetate. With the radial distribution of pairs, it was possible to analyse how the cation interacts with the anion and how the cation interacts with other cations. Thus, it is important to note that similar characteristic liquid phase is present in the system, considering the interatomic distances present. There is also the presence of ion pair formation and possible formation of clusters. These results can contribute to help the different applications of ionic liquids.

\section{References}

[1] Fumino K, Wulf A, Ludwig R and Angew R 2008 Chemie Int Ed. 473830

[2] Herrera C, García G, Atilhan M and Aparicio S A 2016 J. Mol. Liq. 213201 
[3] Shimizu K, Tariq M, Freitas A A, Padua A A H and Lopes J N C 2016 J. Braz. Chem. Soc. 27349

[4] Nasrabadi A T and Gelb L D 2017 J. Phys. Chem. B 121 1908

[5] Perez-Blanco M E and Maginn E J 2011 J. Phys. Chem. B 11510488

[6] Perkins S L, Painter P and Colina C M 2013 J. Phys. Chem. B 11710250
[7] Margulis C J, Stern H A and Berne B J 2002 J. Phys. Chem. B 10612017

[8] Yee P, Shah J K and Maginn E J 2013 J. Phys. Chem. B 117 12556

[9] Reddy T D N and Mallik B S 2017 Phys. Chem. Chem. Phys. 201710358

[10] Thummuru D N R and Mallik B S 2017 J. Phys. Chem. A 2017 8097 